\title{
Acidente Vascular Cerebral Isquêmico com Efeito de Massa
}

\author{
Roberto de Magalhães Carneiro de Oliveira*
}

\section{RESUMO}

Os infartos cerebrais hemisféricos extensos são responsáveis por evolução desfavorável na maioria dos casos de acidente vascular cerebral. Apresentamos uma revisão sobre os mecanismos envolvidos nos infartos cerebrais com grande efeito de massa, os métodos disponíveis para um diagnóstico precoce e para a monitorização adequada e as alternativas de tratamento.

\section{UNITERMOS}

Infarto hemisférico, efeito de massa, tratamento.
Neurologista do Setor de Doenças Neurovasculares da Disciplina de Neurologia da Escola Paulista de Medicina - Unifesp. Neurologista do Setor de Urgências do Complexo Hospitalar São Luiz.

\section{INTRODUÇÃO}

Infartos extensos comprometendo todo o território da artéria cerebral média (ACM), com risco de vida, ocorrem em mais de $10 \%$ dos infartos cerebrais ${ }^{1}$. Geralmente estão associados ao edema cerebral pós-isquêmico, aumento da pressão intracraniana (PIC), piora clínica, coma e morte. O rebaixamento no nível de consciência é um sinal clínico precoce, freqüentemente com assimetria de pupilas e padrão ventilatório periódico com elevação e queda no volume e freqüência respiratórios. O edema em infarto infratentorial, particularmente do cerebelo, é grave, podendo acarretar compressão direta do tronco cerebral.

\section{FISIOPATOLOGIA}

O edema cerebral tem uma natureza bifásica. Inicialmente, a falência nas bombas de sódio, dependentes de energia, leva ao edema intracelular pelo acúmulo de sódio e água dentro da célula. Com a reperfusão do sangue e a quebra da barreira hematoencefálica no tecido isquêmico ocorre edema extracelular, pelo acúmulo local de proteínas plasmáticas e celulares. Com a progressão do edema há deslocamento das estruturas encefálicas através da linha média. Muitas vezes o volume do edema não é compensado pela acomodação de outras estruturas, resultando em elevação da PIC. As alterações no tecido isquêmico e a elevação da PIC serão responsáveis pelo declínio neurológico do paciente.

Menos de 50\% dos pacientes têm elevação da PIC logo no início e, assim, maior mortalidade. Entre aqueles que morrem na fase subaguda do acidente vascular cerebral (AVC), a elevação da PIC é a via final comum. O rebaixamento do nível de consciência, sinal precoce de deterioração neurológica, não é sempre devido ao aumento da PIC com hipoperfusão cerebral. Nos pacientes sem elevação global da PIC, existe clara correlação entre o rebaixamento do nível de consciência e o grau de deslocamento das estruturas pela linha média (glândula pineal). O mecanismo envolvido pode ser a distorção do tronco cerebral, disfunção hemisférica bilateral, ou a compressão 
vascular pelo deslocamento das estruturas. O deslocamento da glândula pineal, entre $4 \mathrm{~mm}$ e $6 \mathrm{~mm}$, da linha média, está associado à sonolência; entre $6 \mathrm{~mm}$ e $8 \mathrm{~mm}$ ao estupor, e maior que $8,5 \mathrm{~mm}$ ao coma ${ }^{2}$. Nos infartos cerebelares a piora clínica pode ser mais rápida, em poucas horas, por 2 mecanismos: o edema cerebelar pode obstruir a passagem do liquor (LCR) pelo IV ventrículo levando à hidrocefalia aguda e à hipertensão intracraniana (HIC), ou causar compressão direta do tronco cerebral.

\section{DIAGNÓSTICO}

\section{Quadro clínico}

Déficits neurológicos graves, como hemiplegia e hemianestesia completas, hemianopsia, desvio forçado do olhar e da cabeça, afasia global, e rebaixamento do nível de consciência são encontrados mais comumente em pacientes com infarto por oclusão da ACM do que em outros tipos de infarto. Rebaixamento do nível de consciência, hemianopsia e infarto em território de toda ACM nos exames diagnósticos de imagem são fatores preditivos independentes de morte ou de grave seqüela. Os pacientes que morrem por edema cerebral são mais jovens do que os que morrem por outras causas, além de morrerem mais rápido (em média 5 dias). Aqueles que desenvolvem coma no primeiro dia têm maior chance de morrer ${ }^{3}$. Escores na escala de AVC do National Institutes of Health $(\mathrm{NIH})>20 \mathrm{mmHg}$ em infartos hemisféricos à esquerda e $>15 \mathrm{mmHg}$ à direita nas primeiras 6 horas de infarto, assim como a presença de náuseas e vômitos nas primeiras 24 horas do AVC e a pressão arterial em 12 horas $>180 \mathrm{mmHg}$ estão associados independentemente ao edema cerebral fatal ${ }^{4}$. Todos esses sinais têm importância na decisão precoce de eventual terapêutica mais intervencionista, antes da irreversibilidade do quadro.

Os pacientes com infarto hemisférico evoluem com progressiva deterioração do nível de consciência nos primeiros 2 dias. Depois disso, sintomas de herniação transtentorial ocorrem entre 2 e 4 dias do início do AVC 5 . Havendo infarto em fossa posterior, podem ocorrer sintomas e sinais cerebelares e de pares cranianos, muitas vezes com rebaixamento do nível de consciência em questão de horas.

As principais causas de infarto cerebral extenso são eventos cardioembólicos, oclusão da artéria carótida interna e dissecção da artéria carótida interna ${ }^{6}$.

\section{Tomografia computadorizada de crânio}

A tomografia computadorizada de crânio (TCC) tem sido analisada em fases mais precoces dos infartos. Os protocolos de trombólise contribuíram muito para a detecção de alterações precoces de infartos extensos ${ }^{7}$. Os principais achados à TCC precoce $(<6$ horas $)$ em pacientes com infarto hemisférico são hipodensidade focal, sinais de edema focal (apagamento de sulcos corticais, compressão de ventrículos e deslocamento de estruturas da linha média) e hiperdensidade da ACM, indicando trombose (Figura 1A). Quanto mais precoce o edema mais desfavorável deve ser a evolução ${ }^{8}$. Sinal de oclusão da bifurcação intracraniana da carótida (oclusão da carótida em " $T$ ") também constitui importante fator preditivo de má evolução ${ }^{9}$. Se a hipodensidade precoce ocupa mais de $50 \%$ do território da ACM, a taxa de mortalidade é bastante elevada (até 85\%) (Figura 2). A presença da hipodensidade na TCC está relacionada ao aumento do conteúdo de água, provavelmente refletindo o edema vasogênico ${ }^{10}$. Pacientes apresentando piora do quadro clínico devem repetir a TCC para avaliar o grau de deslocamento das estruturas encefálicas pelo edema (Figura 1B), ou uma eventual transformação hemorrágica.

\section{Ressonância magnética}

A ressonância magnética cerebral também tem contribuído para um diagnóstico melhor e mais precoce da extensão da isquemia cerebral. Nesse sentido, o estudo da RM ponderada em difusão, ou simplesmente RM por difusão, tem se destacado. O edema citotóxico causa uma restrição da difusão microscópica de prótons. $\mathrm{Na}$ RM por difusão, essa diminuição da difusão de água é presumivelmente refletida na diminuição do coeficiente de difusão aparente (CDA) e é visualizado como um hipersinal. O comportamento do edema citotóxico nos primeiros dias do infarto também pode ser analisado pelo CDA relativo ${ }^{11}$. Em seres humanos, alterações isquêmicas podem ser detectadas pela RM por difusão de $2 \mathrm{a}$ 6 horas após o início dos sintomas ${ }^{12,13}$. Existe boa correlação entre a dimensão da lesão medida na RM por difusão e a evolução clínica ${ }^{14}$. O estudo da perfusão por RM é útil na avaliação inicial do AVC, sendo uma técnica não-invasiva relativamente rápida para documentar uma redução local de fluxo sangüíneo cerebral (FSC).

\section{Monitorização da PIC}

Um infarto extenso com edema, deslocamento da linha média, e o rebaixamento do nível de consciência 

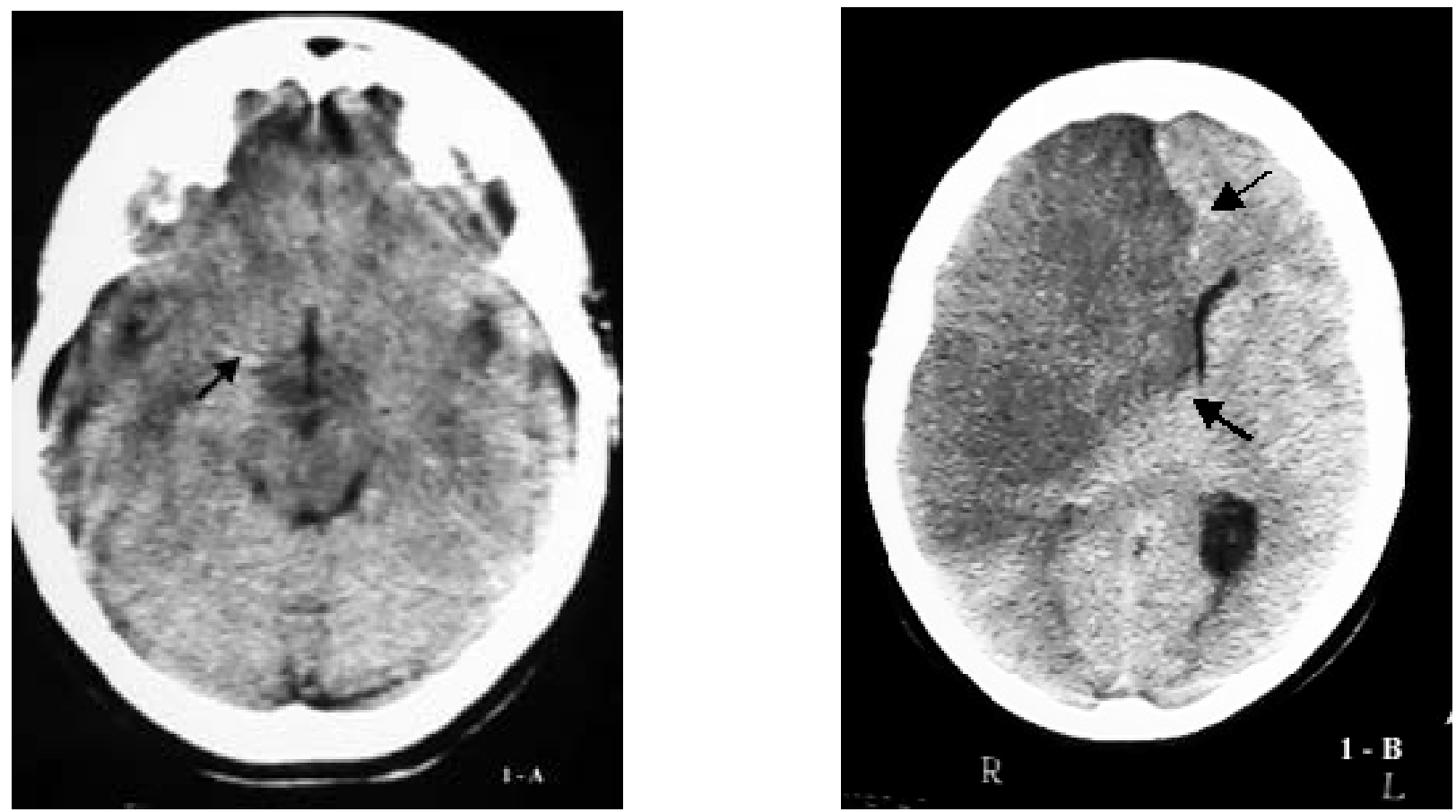

\section{FIGURA1}

Imagem de tomografia computadorizada de crânio em corte axial, mostrando trombose da artéria cerebral média direita (seta) com 3 horas de evolução (A), edema e deslocamento das estruturas pela linha média, após 18 horas de evolução (B).

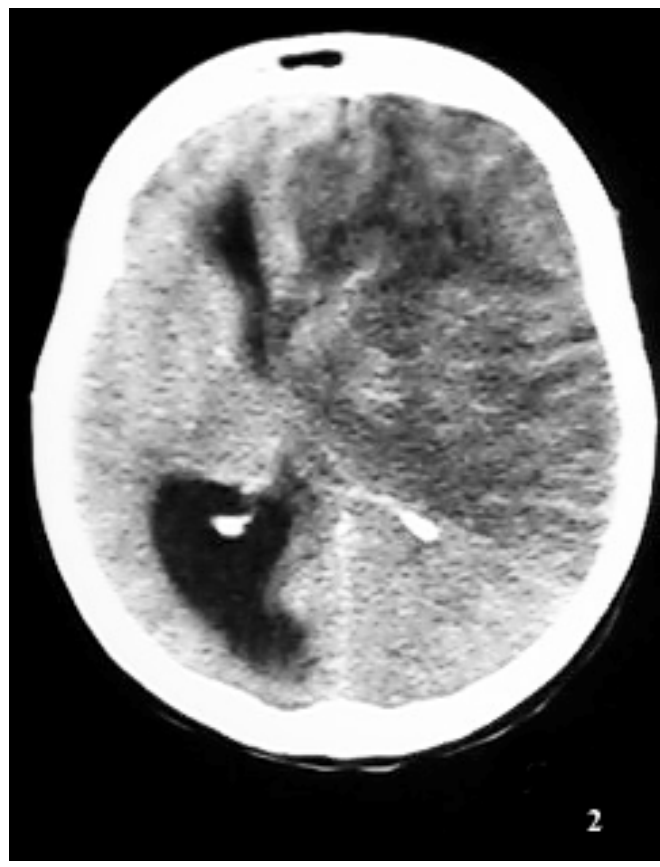

\section{FIGURA2}

Imagem de tomografia computadorizada de crânio em corte axial, com extensa área hipointensa frontoparietal esquerda, com efeito de massa, comprimindo o ventrículo lateral esquerdo e desviando as estruturas da linha média. 
não necessariamente significam que a PIC esteja elevada globalmente. O método de monitorização da PIC que se mantém como "padrão-ouro", desde que foi descrito em 1951, é a medida da pressão do LCR intraventricular. Os cateteres intraventriculares têm a vantagem de possibilitar a drenagem do LCR como modo de controlar a PIC. Por outro lado, têm a desvantagem de ser os mais invasivos e de difícil colocação em pacientes com ventrículos reduzidos. A monitorização da PIC pode ser feita, também, com transdutor epidural ou intraparenquimatoso. Devemos indicar a monitorização da pressão intracraniana nas seguintes situações: a) TCC com lesão extensa e com resposta motora de descerebração e/ou de decorticação (HIC em 70\%); b) pacientes sem abertura ocular, sem resposta verbal e postura motora inadequada (Glasgow <7); c) pacientes com lesão extensa de fossa posterior evoluindo com rebaixamento do nível de consciência e sob risco de hidrocefalia. São contra-indicações para a monitorização da PIC: as coagulopatias e pacientes que estejam conscientes.

A monitorização da PIC, em pacientes com infarto cerebral extenso em território da ACM e com HIC, pode prever a evolução, mas não traz vantagens em termos de sobrevida ou redução de seqüelas ${ }^{15}$. Pacientes com PIC > $35 \mathrm{mmHg}$, em algum momento do tratamento, tendem a evoluir para o óbito. O mau resultado não é um efeito da monitorização da PIC mas uma conseqüência da ineficácia das medidas terapêuticas. A presença de HIC é um importante fator prognóstico e o uso de terapias para reduzir a PIC, baseados unicamente em exames de imagem, sem a devida monitorização, pode ser prejudicial ao paciente.

A monitorização da PIC tem duas graves complicações potenciais: a hemorragia e a infecção. Devemos estar atentos a fatores extracranianos que podem alterar a PIC, e que, se ignorados, podem conduzir-nos a atitudes inadequadas. Algumas dessas situações que exacerbam o aumento da PIC são: a) erro na calibração dos transdutores e monitores da PIC e da pressão arterial (PA); b) obstrução de veias do pescoço (posição inadequada da cabeça e do pescoço, uso de fitas ao redor do pescoço); c) obstrução de vias aéreas (PEEP inadequado, secreções, broncoespasmo); d) relaxamento inadequado dos músculos (espasmos musculares, respiração contra ventilador); e) hipoxia ou hipercapnia; f) novas lesões com efeito de massa (refazer TCC); g) analgesia incompleta, anestesia ou sedação incompletas; h) convulsões; i) febre; j) drogas vasodilatadoras cerebrais; 1) hipovolemia; $m$ ) hiponatremia (freqüentemente por iatrogenia com oferta excessiva de líquidos).

\section{Doppler transcraniano}

Pode orientar como está o FSC, a resposta do FSC às medidas para corrigir a $\mathrm{HIC}$, a presença de vasoespasmo, etc.

\section{Conteúdo venoso de oxigênio}

A diferença de conteúdo de oxigênio arteriovenoso normalmente é de $5 \mathrm{ml} / \mathrm{dl}$ a $7 \mathrm{ml} / \mathrm{dl}$. Valores abaixo de $4 \mathrm{ml} / \mathrm{dl}$ indicam hiperemia cerebral, enquanto valores acima de $9 \mathrm{ml} / \mathrm{dl}$ indicam isquemia cerebral difusa. A saturação de oxigênio no bulbo jugular pode ser monitorizada, preferencialmente de modo contínuo, pois uma única medida é de pouco valor diante das flutuações durante o dia. Tratamentos habituais, que potencialmente provocam isquemia cerebral, podem ser monitorizados por esse método. Hiperventilação e uso de barbitúricos causando queda na PIC, também provocam queda na pressão de perfusão cerebral (PPC), sendo contraproducente em alguns pacientes.

\section{Atividade elétrica cerebral}

A monitorização eletroencefalográfica é útil na decisão sobre o uso de agentes depressores do metabolismo cerebral.

\section{TRATAME NTO}

O tratamento do infarto com efeito de massa deve considerar fatores como: intensidade do edema, tempo de AVC, idade do paciente, local do infarto e grau de complacência cerebral. Não podemos imaginar um tratamento único para os diferentes AVCs com efeito de massa. Basta imaginarmos que é completamente distinta a dinâmica de distribuição da pressão na fossa média e na fossa posterior.

\section{Medidas emergenciais}

Pacientes com deterioração rápida (Glasgow < 9) ou necessitando de manobras de reanimação devem ser entubados e, ventilados de modo adequado, realizando-se rapidamente a TCC para diagnóstico. Pode-se utilizar manitol ( $0,5 \mathrm{mg} / \mathrm{kg}$ em 15 minutos $)$, se houver alteração de reflexo pupilar.

\section{Controle pós-emergencial}

É importante identificar a HIC, evitar ou corrigir os fatores agravantes para que, finalmente, se inicie o 
tratamento fortemente apoiado no conhecimento dos mecanismos envolvidos. Alguns fatores podem agravar o edema cerebral e aumentar a PIC: decúbito baixo (cuidado nos casos de estenose com fluxo limítrofe), $\mathrm{pO}_{2}$ $<50 \mathrm{mmHg}, \mathrm{pCO}_{2}>45 \mathrm{mmHg}, \mathrm{pH}<7,25 \mathrm{e} \mathrm{Na}^{+}<130$ $\mathrm{mmol} / \mathrm{l}$. Havendo indicação, instalar monitorização da PIC.

\section{Medidas gerais}

Alterações metabólicas, infecciosas, crises convulsivas e o uso inadequado de medicações podem agravar o quadro neurológico em pacientes com infarto extenso. O objetivo é manter uma pressão de perfusão cerebral adequada. A PPC é a diferença entre a pressão arterial média e a PIC. A PPC deve estar entre $50 \mathrm{mmHg}$ e 60 $\mathrm{mmHg}$. Abaixo de $30 \mathrm{mmHg}$ pode ocorrer intensa isquemia global.

A) Posição da cabeça. Deve-se proceder à elevação do decúbito de $15^{\circ}$ a $30^{\circ}$ naqueles casos de infarto extenso em que o mecanismo não seja por hipofluxo. As veias de drenagem não devem estar obstruídas por posição inadequada da cabeça ou por agentes compressivos externos. O grau de elevação do decúbito deve ser analisado individualmente, pois alguns pacientes podem apresentar aumento da PIC com a cabeça mais elevada ${ }^{16}$.

B) Ventilação assistida. Pacientes com rebaixamento do nível de consciência necessitam de entubação endotraqueal e ventilação mecânica assistida. Nos infartos hemisféricos, três condições habitualmente indicam a ventilação assistida: crises convulsivas generalizadas tônico-clônicas, edema cerebral e edema pulmonar bilateral. A evolução desses pacientes depende de pronto atendimento e controle da causa subjacente ${ }^{17}$.

C) Volemia. A hipovolemia pode agravar a isquemia por hipotensão e deve ser evitada. Circulação adequada deve ser mantida, se necessário com o uso de colóides. Soluções hipotônicas endovenosas não devem ser aplicadas, mesmo como diluentes de medicamentos.

D) Hipertensão arterial. A hipertensão arterial, a princípio, não deve ser tratada. Havendo necessidade, evitamos o nitroprussiato de sódio, por impedir a auto-regulação vascular intracraniana. Devemos atentar às causas de hipertensão arterial, como dor, repleção vesical e sedação inadequada.

E) Distúrbios hidroeletrolíticos. Devem ser corrigidos.

F) Convulsões. Devem ser evitadas. Podem ser dificilmente reconhecidas se o paciente estiver entubado e sob ventilação assistida. Episódios de dilatação pupilar, elevação da PA e da PIC são sugestivos.
G) Temperatura. A febre aumenta o metabolismo cerebral, promove vasodilatação cerebral e agrava o edema. Deve ser prevenida e combatida, de preferência com acetaminofen ou ácido acetilsalicílico. Alguns centros têm desenvolvido o controle da HIC em pacientes com infarto extenso pela hipotermia moderada $\left(\mathrm{T}=33^{\circ} \mathrm{C}\right)$, com bons resultados ${ }^{18}$.

H) Hiperglicemia. A hiperglicemia deve ser evitada. A isquemia cerebral e o infarto são agravados por hiperglicemia, com progressão do edema cerebral e aumento da PIC.

\section{Hiperventilação}

A hiperventilação induz a hipocarbia e faz baixar a PIC pela redução no volume sangüíneo cerebral. O efeito é quase imediato, mas as reduções máximas podem demorar de 15 a 30 minutos após a modificação da $\mathrm{pCO}_{2}$. $\mathrm{O}$ efeito dura aproximadamente 1 dia, pelos mecanismos compensadores do bicarbonato dentro do cérebro e das células musculares lisas dos vasos, corrigindo o $\mathrm{pH}$ intra $\mathrm{e}$ extracelular. Pode haver efeito rebote ao se suspender subitamente a hiperventilação. O método ideal é aumentar a freqüência no ventilador, mantendo-se um volume corrente de $12 \mathrm{ml} / \mathrm{kg}$ a $15 \mathrm{ml} / \mathrm{kg}$ de peso. $\mathrm{A} \mathrm{pCO}_{2}$ deve ser reduzida para $25 \mathrm{mmHg}$ a $35 \mathrm{mmHg}$. Quando a hiperventilação deixa de ser desejada (PIC constante $<15 \mathrm{mmHg}$ ), a retirada sistemática deve ser feita num período de 12 a 24 horas. Apenas as áreas normais respondem à hiperventilação. As pressões diminuídas do enchimento cardíaco, em virtude da ventilação com pressão positiva, podem ser agravadas pela hiperventilação. Como resultado, o benefício da hiperventilação com redução da PIC pode ser eliminado pela hipotensão e perfusão cerebral comprometida. Corrige-se esse problema mantendo um bom volume intravascular (com hiperosmolaridade). Hiperventilação excessiva pode precipitar isquemia cerebral, com alentecimento da atividade elétrica cerebral, acidose láctica liquórica e aumento da diferença de conteúdo arteriovenoso de oxigênio > $9 \mathrm{ml} / \mathrm{dl}$. Estudos do FSC por TC com xenônio, em pacientes com lesão cerebral aguda submetidos à hiperventilação, concluíram que esta pode induzir à isquemia cerebral nas áreas lesadas, assim como nas áreas normais ${ }^{19}$. O doppler transcraniano é um método simples e seguro para avaliar a reatividade ao $\mathrm{CO}_{2}$.

\section{Agentes hiperosmolares}

Reduzem a PIC e aumentam a complacência intracraniana por vasoconstrição cerebral, reduzindo a incidência e a gravidade das ondas em platô. 
A) Glicerol: álcool hidrossolúvel metabolizado no organismo por ação da enzima gliceroquinase, presente inclusive no cérebro. Provoca aumento imediato na osmolaridade plasmática e queda na pressão intracraniana. A dose recomendada é de $1,5 \mathrm{mg} / \mathrm{kg} / \mathrm{dia}$, diluído a $10 \%$, dividido em 3 doses diárias, cada uma delas aplicada em 4 horas. Se aplicado por via endovenosa, começa a atuar em 10 minutos e em 20 minutos provoca acentuada queda na PIC. Pode ser administrado por via oral ou endovenosa. Não provoca rebote, pois ao atravessar o endotélio é metabolizado no interstício. Apresenta baixa toxicidade e boa tolerabilidade. O glicerol, em pacientes com infarto cerebral extenso, mostra-se eficaz em reduzir o edema cerebral e melhorar o prognóstico ${ }^{20}$.

B) Manitol: aumenta a osmolaridade plasmática com desidratação, expansão plasmática e hemodiluição. Melhora o FSC e mantém a microcirculação patente. Atua melhor nas fases iniciais do edema isquêmico de quadros moderados e na zona de penumbra. Pode causar efeito rebote. O início da ação ocorre após 10 a 30 minutos, e o pico, após 20 a 60 minutos. A dose é de $0,75 \mathrm{~g} / \mathrm{kg}$ a $1 \mathrm{~g} / \mathrm{kg}$ seguida de $0,25 \mathrm{~g} / \mathrm{kg}$ a $0,5 \mathrm{~g} / \mathrm{kg}$ a cada 3 a 5 horas. Devemos buscar uma osmolaridade alvo de $300 \mathrm{mOsm} / 1$.

São desvantagens dos agentes hiperosmolares: desequilíbrio hidroeletrolítico (hipocalemia, alcalose hipoclorêmica, hiperosmolaridade sérica "maligna" com lesão renal); hiperglicemia (naqueles usando glicerol); hipertensão abrupta, aumento no volume sangüíneo cerebral, propiciando herniação cerebral (quando de uso excessivo e rápido); e aumento da PIC (nas zonas com quebra da barreira hematoencefálica, pela passagem dos agentes hiperosmolares para o tecido perivascular).

\section{Diuréticos}

Podem reduzir a PIC pela produção de gradiente osmótico na direção do espaço intravascular pela ligeira diurese osmótica, redução da produção de LCR, e pela remoção de sódio e água do cérebro normal edemaciado. Deve-se evitar hipotensão e hipovolemia, além de checar os eletrólitos sangüíneos.

\section{Barbitúricos}

Reduzem o metabolismo nas regiões não afetadas e, assim, reduzem a PIC; reduzem o edema cerebral vasogênico e promovem a varredura de radicais livres ${ }^{21}$. Devem ser utilizados quando a PIC não é controlada por terapias convencionais. Os mais usados são o tiopental $(1 \mathrm{mg} / \mathrm{kg}$ a $5 \mathrm{mg} / \mathrm{kg})$ e o pentobarbital. O pentobarbital é o mais adequado para controle prolongado da PIC, com meia-vida de aproximadamente 24 horas. Sua ação é rápida, com início de efeito entre 5 e 10 minutos. Devese monitorizar a PIC, PA e FSC (por doppler transcraniano, se possível). Apresentam como complicações: impossibilidade do exame neurológico adequado, depressão miocárdica, hipotensão arterial e aumento do risco de pneumonia e sépsis. Deve-se dar atenção ao grau de hidratação e, se necessário, usar agentes inotrópicos (dobutamina). O cateter de Swan-Ganz é útil na avaliação da função miocárdica. O tiopental $(5 \mathrm{mg} /$ $\mathrm{kg} / 15$ minutos) em pacientes com infarto hemisférico pode baixar a PIC de modo eficiente, mas não altera o resultado neurológico ${ }^{21}$.

\section{Corticóides}

Reduzem edema vasogênico mas não trazem qualquer benefício no controle da PIC em pacientes com infarto cerebral. Podem levar à hiperglicemia com piora do edema, têm potencial trombogênico e facilitam o aparecimento de infecções. Não devem ser usados.

\section{Intervenção cirúrgica}

Deve ser analisada como medida heróica, salvadora, e necessita de perfeita integração entre as equipes clínica e cirúrgica. Temos duas categorias de estratégia cirúrgica, aquelas que ressecam o tecido cerebral edematoso e as que não o fazem. A delimitação exata do tecido cerebral não viável é muito difícil. Classicamente, a lobectomia temporal ou frontal tem sido utilizada, especialmente no hemisfério não dominante. A dilatação ventricular aguda demanda imediata drenagem ventricular e deve ser bilateral, se a lesão for de linha média. Pacientes com infarto extenso, evoluindo com HIC e sinais de herniação, apresentam melhora após craniotomia descompressiva ${ }^{22,23}$. O momento da indicação cirúrgica usualmente ocorre quando se verifica a falência de todas as outras formas de conter a HIC e uma eventual herniação, quando, muitas vezes, o infarto já assumiu proporções irreversíveis. A indicação precoce de hemicraniectomia (nas primeiras 24 horas do AVC) em pacientes com sinais de infarto extenso à TCC inicial, antes de haver deterioração clínica, mostra resultados melhores que o tratamento conservador ${ }^{24}$. O procedimento deve ser realizado tão logo indicado. A craniotomia extensa permite melhor protrusão do tecido edemaciado e diminui as chances de compressão de 
estruturas vasculares na borda do osso, com conseqüente piora do infarto. A abertura da dura-máter é essencial para a expansão adequada do tecido edemaciado. Pacientes com infarto cerebelar extenso e hidrocefalia, com preservação da consciência, podem ser tratados inicialmente com drenagem ventricular externa ${ }^{25}$. Havendo deterioração progressiva do quadro clínico, a craniotomia suboccipital descompressiva e imediata pode ser extremamente benéfica ${ }^{26}$.

\section{SUMMARY}

Ischemic stroke with mass effect.

Hemispheric strokes are often responsible for critical evolution. We present a review of physiopathology, clinical presentation, diagnosis and management of ischemic stroke with mass effect.

\section{KEYWORDS}

Hemispheric stroke, mass effect, management.

\section{Referências}

1. Silver FL, Norris JW, Lewis AJ, Hachinski VC. Early mortality following stroke: a prospective review. Stroke, 15:492-6, 1984.

2. Ropper AH. Lateral displacement of the brain and level of consciousness in patients with na acute hemispheral mass. N Engl J Med, 314:953-8, 1986.

3. Heinsius T, Bogousslavsky J, Van Melle G. Large infarcts in the middle cerebral artery territory. Etiology and outcome patterns. Neurology, 50:341-50, 1998.

4. Krieger DW, Demchuk AM, Kasner SE, Jauss M, Hantson L. Early clinical and radiological predictors of fatal brain swelling in ischemic stroke. Stroke, 30:287-92, 1999.

5. Hacke W, Schwab S, Horn M, Spranger M, De Georgia M, von Kummer R. Malignant middle cerebral artery territory infarction: clinical course and prognostic signs. Arch Neurol, 53:309-15, 1996.

6. Bingaman WE, Frank JI. Malignant cerebral edema and intracranial hypertension. Neurol Clin, 13:479-509, 1995.

7. Von Kummer R, Allen KL, Holle R, Bozzao L, Bastianello S, Manelfe C, Bluhmki E, Ringleb P, Meier DH, Hacke W. Acute stroke: usefulness of early CT findings before thrombolytic therapy. Radiology, 205:327-33, 1997.

8. Büttner T, Uffmann M, Günes N, Köster O. Early CCT signs of supratentorial brain infarction: clinico-radiological correlations. Acta Neurol Scand, 96:317-23, 1997.

9. Kucinski T, Koch C, Grzyska U, Freitag HJ, Krömer H, Zeumer $\mathrm{H}$. The preditive value of early $\mathrm{CT}$ and angiography for fatal hemispheric swelling in acute stroke. Am J Neuroradiol, 19:839-46, 1998.

10. Unger E, Littlefield J, Gado N. Water content and water structure in CT and MR signal changes: possible influence in detection of early stroke. Am J Neuroradiol, 9:687-91, 1988.

11. Schlaug G, Sieewrt B, Benfield A, Edelman RR, Warach S. Time course of the apparent diffusion coeficient (ADC) abnormality in human stroke. Neurology, 49:113-9, 1997.
12. Sorensen AG, Buonanno FS, Gonzalez RG, Schwamm LH, Lev MH, Huang Hellinger FR, Reese TG, Weisskoff RM, Davis TL, Suwanwela N, Can U, Moreira JA, Copen WA, Look RB, Finklestein SP, Rosen BR, Koroshetz WJ. Hyperacute stroke: evaluation with combined mutisection diffusion-weighted and hemodinamically weighted echo-planar MR imaging. Radiology, 199:391-401, 1996.

13. Warach S, Gaa J, Siewart B, Wiclopolski P, Edelman RR. Acute human stroke studied by whole brain echoplanar diffusion-weighted magnetic resonance imaging. Ann Neurol, 37:231-41, 1995.

14. Van Everdingen KJ, van der Grond J, Kappelle LJ, Ramos LMP, Mali WPTM. Diffusion-weighted magnetic resonance imaging in acute stroke. Stroke, 29:1783-90, 1998.

15. Schwab S, Aschoff A, Spranger M, Albert F, Hacke W. The value of intracranial pressure monitoring in acute hemispheric stroke. Neurology, 47:393-8, 1996.

16. Ropper AH, O'Rourke D, Kennedy SK. Head position, intracranial pressure, and compliance. Neurology, 32:1288-91, 1982.

17. Wijdicks EFM, Scott JP. Causes and outcome of mechanical ventilation in patients with hemispheric ischemic stroke. Mayo Clin Proc, 72:210-3, 1997.

18. Schwab S, Schwarz S, Spranger M, Keller E, Bertram M, Hacke W. Moderate hypothermia in the treatment of patients with severe middle cerebral artery infarction. Stroke, 29:2461-6, 1998.

19. Stringer WA, Hasso NA, Thompson JR, Hinshaw DB, Jordan KG. Hyperventilation-induced cerebral ischemia in patients with acute brain lesions: demonstration by xenon-enhaced CT. Am J Neuroradiol, 14:475-84, 1993.

20. Meyer JS, Charney JZ, Rivera VM, Mathew NT. Treatment with glycerol of cerebral edema due to acute cerebral infarction. Lancet, 7732:993-7, 1971.

21. Schwab S, Spranger M, Schwarz S, Hacke W. Barbiturate coma in severe hemispheric stroke: useful or obsolete? Neurology, 48:1608-13, 1997.

22. Kondziolka D, Fazl M. Functional recovery after decompressive craniectomy for cerebral infarction. Neurosurgery, 23:143-7, 1988.

23. Delashaw JB, Broaddus WC, Kassell NF, Haley EC, Pendleton GA, Vollmer DG, Maggio WW, Grady MS. Treatment of right cerebral infarction by hemicraniectomy. Stroke, 21:874-81, 1990.

24. Schwab S, Steiner T, Aschoff A, Schwarz S, Steiner HH, Jansen O, Hacke W. Early hemicraniectomy in patients with complete middle cerebral artery infarction. Stroke, 29:188893, 1998.

25. Mathew P, Teasdale G, Bannan A, Oluoch-Olunya D. Neurosurgical management of cerebellar haematoma and infarct. J Neurol Neurosurg Psychyatry, 59:287-92, 1995.

26. Chen HJ, Lee TC, Wei CP. Treatment of cerebellar infarction by decompressive suboccipital craniectomy. Stroke, 23:95761, 1992.

\section{Endereço para correspondência:} Roberto de M. Carneiro de Oliveira Rua Desembargador Aguiar Valim, 230 CEP 04535-100 - São Paulo, SP E-mail:rmco@ig.com.br 